\title{
Chancen für Frauen in der Wissenschaft - eine Frage der Fachkultur? - Retrospektive Verlaufsanalysen und aktuelle Forschungsergebnisse
}

\section{Inken Lind und Andrea Löther}

Im vorliegenden Beitrag wird erstmals die Chance auf eine wissenschaftliche Laufbahn über einen 18jährigen Qualifikationsverlauf für beide Geschlechter getrennt ausgewiesen. Einleitend wird die Methodik der retrospektiven Verlaufsanalyse vorgestellt. Die Ergebnisse verdeutlichen die ungleiche Partizipation der Frauen am wissenschaftlichen Qualifikationsprozess, die sich nicht auf eine geringe Bildungsbeteiligung zurückführen lässt. Bei der fächerspezifischen Betrachtung werden die teilweise großen Unterschiede zwischen den einzelnen Disziplinen deutlich, die bislang noch wenig zur Kenntnis genommen wurden. Die retrospektive Verlaufsanalyse wird ergänzt durch aktuelle Erkenntnisse aus dem Forschungsfeld zu Barrieren für Frauen in der Wissenschaft und der Wirkung verschiedener Fachkulturen. Auf der Grundlage der dargestellten Ergebnisse werden verbreitete Annabmen zu den Ursachen des geringen Frauenanteils in der Wissenschaft kritisch hinterfragt und neue Perspektiven für einen weiterführenden Diskurs und Forschungsdesiderata aufgezeigt.

Die aktuelle Diskussion um die Situation von Frauen in der Wissenschaft ist geprägt von der Frage, warum in Deutschland trotz aller hochschulpolitischer Bemühungen um Frauenförderung und Gleichstellung Frauen deutlich seltener ihre Qualifikation in eine Wissenschaftskarriere umsetzen können als Männer und die teilweise positiven Entwicklungen langsamer voranschreiten als in anderen europäischen Ländern.

Die Zahlen verdeutlichen die Situation: Einem Studentinnenanteil von 47,7 Prozent standen 2005 in Deutschland 14,2 Prozent Professorinnen gegenüber. Ähnlich sieht es an österreichischen Universitäten mit einem Professorinnenanteil von 13,9 Prozent aus, bei 53,3 Prozent Studentinnen. In der Schweiz waren 23,6 Prozent der Professuren 2005 mit Frauen besetzt, allerdings nur 12,4 Prozent der Universitätsprofessuren. ${ }^{1}$

Im europäischen Vergleich bilden damit Deutschland und Österreich zusammen mit den Niederlanden und Belgien die Schlusslichter bezüglich des Frauenanteils an Professuren. Finnland hatte 2004 mit 21,2 Prozent einen mehr als 
doppelt so hohen Frauenanteil an den höchsten Positionen an Hochschulen (9,0 bis 9,5 Prozent; European Commission, 2006, S. 57). ${ }^{2}$ Im EU-Durchschnitt lag der Frauenanteil an den höchst dotierten Professuren im Jahr 2004 bei 15,3 Prozent. In Rumänien, Lettland und der Türkei sind sogar mehr als ein Viertel dieser Professuren mit Frauen besetzt.

Zur Erklärung dieses Phänomens werden in der öffentlichen Debatte mitunter Argumente und Ursachenzuschreibungen bemüht, die einer kritischen Betrachtung und fachlichen Überprüfung kaum standhalten. So gehört die Argumentation, der niedrige Frauenanteil an Professuren liege vor allem in der geringeren Bildungsbeteiligung der entsprechenden Altersgruppen begründet, zu den weit verbreiteten Alltagsannahmen innerhalb der Wissenschaft (Holzbecher, 1997). Gleichzeitig bezieht sich die Auseinandersetzung vor allem auf den querschnittlichen Frauenanteil über alle Fächer, während disziplinäre Unterschiede von Qualifikationsverläufen bislang noch keine ausreichende Berücksichtigung fanden. Der Blick auf Professorinnenanteile und die häufig vorgenommene Gegenüberstellung von Professorinnen- und Studentinnenanteil in einem Berichtsjahr gibt zwar einen ersten Einblick in die Geschlechterverhältnisse an Hochschulen, ist für eine Analyse der Ursachen und Wirkungsweisen jedoch nur bedingt geeignet. Größeren Erkenntniswert liefert die Betrachtung des Qualifikationsprozesses im zeitlichen Verlauf. Das CEWS (Kompetenzzentrum Frauen in Wissenschaft und Forschung) analysiert daher das zur Verfügung stehende Datenmaterial unter der Annahme idealtypischer Karriereverläufe. ${ }^{3}$ Bestandsdaten für Professuren sind für eine solche Untersuchung ungeeignet, da in ihnen eine große Anzahl an Altersgruppen mit unterschiedlichen Ausgangsbedingungen hinsichtlich der Beteiligung von Frauen und Männern in der Wissenschaft vereinigt sind. Benötigt werden daher Daten über Neuberufungen, die für Deutschland seit 1997 als Erhebung der Bund-Länder-Kommission für Bildungsplanung und Forschungsförderung (BLK) zur Verfügung stehen.

Der Beitrag gliedert sich in zwei Hauptabschnitte: Zunächst werden geschlechtsspezifisch unterschiedliche Chancen auf wissenschaftliche Qualifikationen und Positionen dargelegt. Diese statistischen Ergebnisse werden ergänzt durch Erkenntnisse der Ursachenforschung zur Marginalisierung von Frauen in der Wissenschaft. Der zweite Teil des Beitrages ist den disziplinären Unterschieden gewidmet. Diese werden anhand ausgewählter Fächergruppen veranschaulicht und in Beziehung zu aktuellen Thesen und Ergebnissen zu den Bedingungsfaktoren gestellt. Im abschließenden Fazit werden verbreitete Annahmen kritisch hinterfragt und weiterführende Perspektiven für den wissenschaftlichen Diskurs und die hochschulpolitische Auseinandersetzung mit dem geringen Frauenanteil in der Wissenschaft aufgezeigt. 


\section{Fächerübergreifende Barrieren in der wissen- schaftlichen Qualifikation von Frauen}

\section{Methodik der retrospektiven Verlaufsanalyse}

Ausgehend von den aktuellen Berufungen bilden wir retroperspektiv einen idealtypischen Karriereverlauf. Die Dauer der einzelnen Qualifikationsschritte stützt sich dabei auf Ergebnisse der Hochschulforschung, Angaben des Statistischen Bundesamtes und gesetzlichen Vorgaben zu Befristungsregelungen. Für die Studiendauer gehen wir von sechs Jahren aus, gestützt auf Angaben des Statistischen Bundesamtes, der HIS-Absolventenstudie von 1992/93 sowie des Wissenschaftsrats (Schaeper \& Minks, 1997; Wissenschaftsrat, 2001; vgl. auch Heublein \& Schwarzenberger, 2005). Die angenommene Promotionsdauer von vier Jahren beruht auf Angaben des Wissenschaftsrats zu Promotionen in Sonderforschungsbereichen (Wissenschaftsrat, 1997). Die Habilitationsdauer von sechs Jahren bezieht sich auf die gesetzlichen Befristungsregelungen für C1Assistentenstellen. ${ }^{4}$ Für den Übergang von der Habilitation in die Professur schließlich werden zwei Jahre angesetzt; dazu fehlen jedoch gesicherte Angaben. Das Statistische Bundesamt gibt als Durchschnittsalter bei der Ernennung auf eine Professur 41,1 Jahre an.

Die medizinischen Fächer weisen mit einer kürzeren Promotionsdauer und einer längeren Qualifikationszeit nach der Promotion einen etwas anderen Qualifikationsverlauf auf. Insgesamt dauert die Qualifikation vom Studienbeginn bis zur Professur jedoch ähnlich lange und aus Gründen der Vergleichbarkeit werden daher für die medizinischen Fächer keine gesonderten zeitlichen Schnitte gesetzt.

Die Berufungen auf eine Fachhochschulprofessur sind in den Daten enthalten. Der besondere Qualifikationsweg wird jedoch nicht gesondert untersucht. Qualifikationsvoraussetzung für eine Fachschulprofessur sind eine Promotion sowie fünfjährige Berufserfahrung, davon mindestens drei Jahre außerhalb der Hochschule. Es ist also keine Habilitation zu erbringen. Aufgrund der Berufstätigkeit unterscheidet sich das Alter bei der Erstberufung auf Fachhochschulprofessuren aber nicht wesentlich von dem für Universitätsprofessuren (nach Angaben des Statistischen Bundesamtes für 2005: 40,8 Jahre gegenüber 41,1 Jahre). Der vorgeschlagene idealtypische Karriereverlauf ist damit auch für diesen Karriereweg vergleichbar.

Insgesamt gehen wir also von einer idealtypischen Qualifikationsdauer zwischen Studienbeginn und Berufung auf eine Professur von 18 Jahren aus. ${ }^{5}$ Es ist jedoch zu beachten, dass es sich in der retrospektiven Analyse um die Untersuchung idealtypischer Karriereverläufe handelt; Daten für empirische Kohortenanalysen von Wissenschaftler/innen stehen für Deutschland leider nicht zur Verfügung.

Grundlage für die Angaben zu Studierenden, Prüfungen und Habilitationen bilden Daten des Statistischen Bundesamtes. Die Daten für Neuberufungen beruhen auf Erhebungen der BLK. ${ }^{6}$ Die Berechnungen wurden durch das CEWS 
durchgeführt. Um Zufallsschwankungen zu vermeiden, werden für Studienabschlüsse, Promotionen, Habilitationen und Berufungen die Daten von jeweils drei Jahren zusammengefasst.

Die Erhebungen der BLK erfassen die Neuberufungen, differenziert nach Geschlecht, Hochschulart, Fächergruppe, Besoldungsgruppe und Bundesland. In der Gesamtzahl der Berufungen sind auch die Wechsel von Professor/innen an eine andere Hochschule enthalten. Für unsere retrospektive Analyse des Karriereverlaufs interessieren als Qualifikationsschritt nach der Habilitation jedoch die Erstberufungen. Da diese Daten nicht gesondert ausgewiesen werden, werten wir in dieser Untersuchung als Näherung die Berufungen auf C2-, C3(ohne Fachhochschulen) und W2-Professuren aus, da wir davon ausgehen, dass es sich hierbei zum überwiegenden Teil um Erstberufungen handelt.

Nach Fächergruppen aufgeschlüsselte Daten liegen nur für Fachhochschulen und Universitäten vor; in den vorliegenden Daten fehlen also Berufungen an Künstlerische Hochschulen. Auf eine Untersuchung der Fächergruppe Kunst/Kunstwissenschaft muss daher verzichtet werden, da das Bild ohne die Berufungen an Künstlerische Hochschulen unvollständig wäre.

Angaben zu den Hochschulberechtigten lassen sich nicht fächergruppenspezifisch auswerten. Diese Daten werden daher nur für die fächerübergreifende Analyse verwendet.

Abgesehen von Deutschland werden nur in Dänemark Rekrutierungsdaten erhoben. Ein europäischer Vergleich dieser Analyse ist daher derzeit nicht möglich.7 Der vorliegende Beitrag zeigt insofern auch den Nutzen von Rekrutierungsdaten für die Bildungsforschung.

\section{Fächerübergreifende Barrieren: Promotion und Habilitation 8}

Fächerübergreifend lag der Frauenanteil an den Berufungen auf C2-/C3-/W2Professuren in den Jahren 2003-2005 bei 22,2 Prozent. Unter den Studienanfängern dieser Kohorte war der Frauenanteil dagegen mit 40,3 Prozent fast doppelt so hoch. Abbildung 1 macht sichtbar, welches die entscheidenden Barrieren für Frauen im Qualifikationsverlauf sind: Im Übergang zur Promotion und erneut im Übergang zur Habilitation fällt der Frauenanteil um jeweils rund 10 Prozentpunkte. Wissenschaftlerinnen, die diese Qualifikation erreicht haben, sind im Übergang zu einer Professur dann genauso erfolgreich wie Männer: Der Frauenanteil an den Berufungen auf C2-/C3/W2-Professuren entspricht dem Anteil an den Habilitationen zwei Jahre zuvor. Schwieriger gestaltet sich dagegen der Aufstieg in die höchsten Positionen an Hochschulen, die C4/W3-Professuren und die C3-Professuren an Fachhochschulen. Ein erster Einbruch ist zudem beim Übergang von der Schule in die Hochschule zu verzeichnen: Frauen mit einer Hochschulberechtigung entschieden sich seltener für ein Studium als Männer, ein Umstand, der auch 2005 mit einem Frauenanteil von 57 Prozent an den Studienberechtigten und 49 Prozent an den Studienanfängern festzustellen ist. 


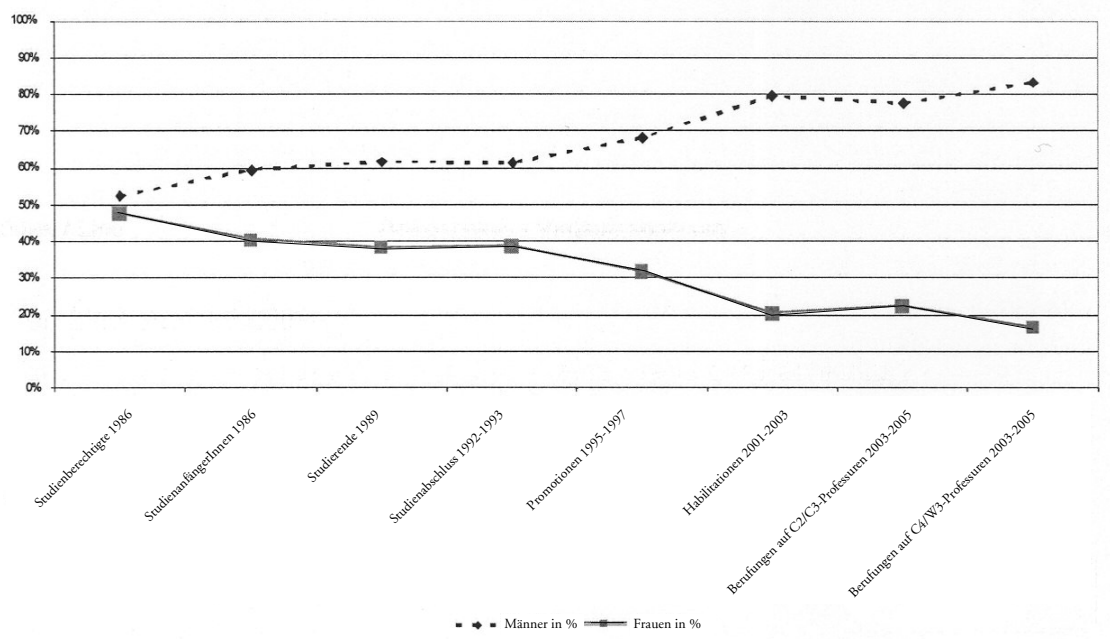

Abbildung 1: Qualifikationsverlauf für alle Fächergruppen, 1986- 2005

Die Frauen- und Männeranteile im idealtypischen Karriereverlauf lassen sich in graphischer Form als eine Schere beschreiben (European Commission, 2006, S. 13). Um zu erkennen, ob sich dieses Muster im zeitlichen Verlauf veränderte, wurden die Daten für die aktuellen Berufungen (2003-2005) mit den ältesten Daten, die für Neuberufungen zur Verfügung stehen (1997-1999), verglichen. Damit konnte der idealtypische Karriereverlauf für diejenigen, die $1980 \mathrm{ihr}$ Studium begannen, mit den Studienanfängern von 1986 verglichen werden.

Die Frauen- und Männeranteile während der Studienphase sind für beide Vergleichsgruppen gleich. Tatsächlich blieb der Studentinnenanteil in Deutschland zwischen 1980 und 1989 fast konstant bei rund 40 Prozent. ${ }^{9}$ Eine geringfügige Veränderung zeigt sich bei den Promotionen und den Habilitationen. Bei fast gleichem Anteil an den Studienabschlüssen 1985-87 und 1991-93 promovierten und habilitierten Frauen der jüngeren Kohorte häufiger als die Gruppe der Studienanfängerinnen von 1980. Der Frauenanteil an den Promotionen stieg um 4 Prozentpunkte, der an den Habilitationen um 6 Prozentpunkte. Im Übergang von der Habilitation zur Professur dagegen scheinen ältere Wissenschaftlerinnen etwas bessere Chancen gehabt zu haben: Bei einem Frauenanteil an den Habilitationen von 14 Prozent lag ihr Anteil an den Berufungen bei 19 Prozent, während die Frauenanteile an Habilitationen und Berufungen bei den jüngeren Wissenschaftlerinnen fast gleich sind. Trotz dieser geringfügigen Unterschiede, die möglicherweise mit verstärkten Anstrengungen in den 1990er Jahren, Frauen zu einer Promotion und Habilitationen zu führen, zu erklären sind, waren die entscheidenden Barrieren für Wissenschaftlerinnen, die 1980 bzw. 1986 ihr Studium begannen, gleich: Mit der Promotion und der Habilitation scheiden deutlich mehr Frauen als Männer aus der Wissenschaft aus. 


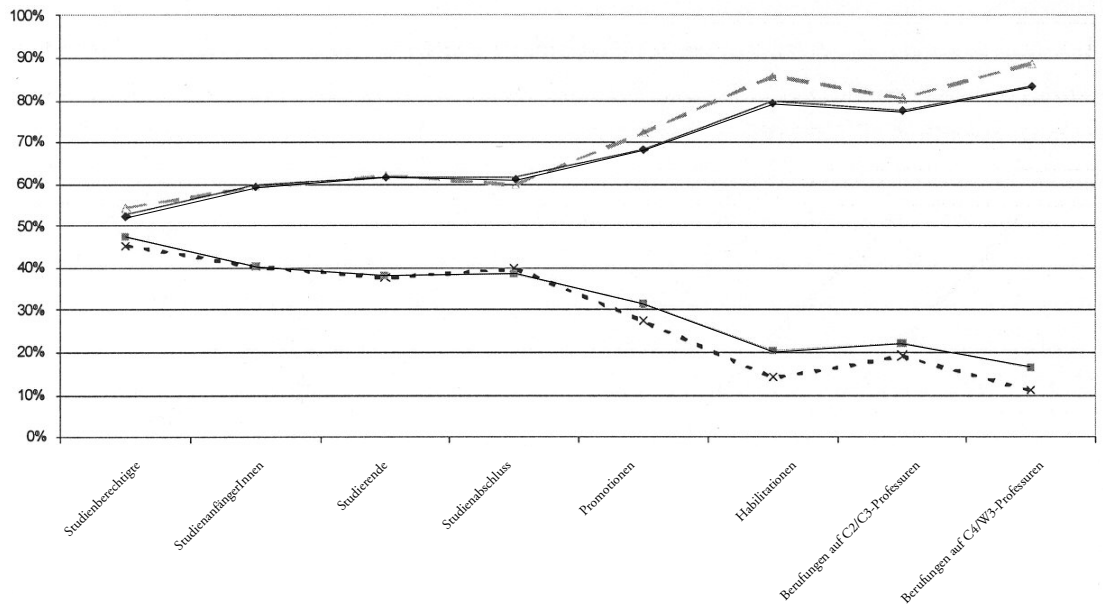

Abbildung 2: Qualifikationswege der Studienanfänger/innen 1980 und 1986 im Vergleich

\section{Erklärungsansätze zu Geschlechterdifferenzen im wissen- schaftlichen Qualifikationsverlauf}

Die Ursachen für das scherenartige Auseinanderdriften im Qualifikationsverlauf und insbesondere im Übergang zwischen Studium und Promotion wurden verstärkt seit den 80 er Jahren untersucht. ${ }^{10}$ Als eine der wesentlichen Ursachen für die geringere Promotionsneigung von Frauen wird heute eine unterschiedliche Bestätigungskultur von Seiten der Hochschullehrenden gegenüber Studentinnen und Studenten benannt (Allmendinger, 2005; Allmendinger et al., 2001; Bochow \& Joas, 1987; Geenen, 1994; Leemann, 2002; Noeres \& Kirschbaum, 2004). Die entsprechenden Studien liefern konsistente Belege, dass Studentinnen und Absolventinnen sich während des Studiums weniger in ihren fachlichen Kompetenzen gestärkt fühlen als ihre Kommilitonen und auch seltener direkt zu einer Promotion aufgefordert werden (Allmendinger, 2005; Bochow \& Joas, 1987; Geenen, 1994; Kirschbaum et al. 2005; Koch, 1995; Leemann, 2002). Geschlechtsspezifische Unterschiede zeigen sich in den Erwartungen, die mit einer Promotion verbunden sind: Studenten stufen den Effekt einer Promotion für die eigene berufliche Laufbahn höher ein als Studentinnen. Daneben erwarten Studenten mehr Anerkennung für eine Promotion vom sozialen Umfeld (Holzbecher et al., 2002; Spies \& Schute, 1999). Schließlich spielen subtile Unterschiede in der Berufsorientierung von Studentinnen und Studenten sowie in der Motivationslage und Selbstwirksamkeitserwartung eine Rolle (Spies \& Schute, 1999; Zimmermann, 1996). 
Wissenschaftliche Laufbahnen von Frauen werden nach wie vor in erster Linie nach der Promotion abgebrochen (Enders, 1996). In Bezug auf die Frage, warum so viele Frauen nach erfolgreichem Abschluss der Promotion die Wissenschaft verlassen, spielt die Situation und das Erleben der Promotionsphase eine zentrale Rolle. Hinsichtlich des beruflichen Status während der Promotion wurde bereits seit den 1980er Jahren ein geringerer formaler Status von Doktorandinnen im Vergleich zu Doktoranden festgestellt (Bochow \& Joas, 1987; Holtkamp et al., 1986; Holzbecher et al., 2002; Koch, 1995; Krimmer \& Zimmer, 2003), der sich auch noch nach der Promotion im wissenschaftlichen Mittelbau fortsetzt (Achatz et al., 2000; Enders \& Teichler, 1995; Hoffmann-Lange, 2000; Wimbauer, 1999).

Allmendinger, Fuchs und v. Stebut (1999) konnten nachweisen, dass der formale Status während der Promotion unmittelbar mit der Austrittswahrscheinlichkeit aus dem Wissenschaftssystem verbunden ist. Bei niedrigerem Status wird die wissenschaftliche Laufbahn nach der Promotion eher abgebrochen; dieser Effekt zeigt sich für beide Geschlechter gleichermaßen. Da aber Frauen einen geringeren formalen Status zu Beginn der wissenschaftlichen Laufbahn innehaben, ist entsprechend ihre Austrittswahrscheinlichkeit höher. Damit geht auch einher, dass Doktorandinnen häufiger auf Stipendien promovieren als Doktoranden. Was sich auf den ersten Blick als Vorteil darstellt, von Lehr- und Institutsverpflichtungen freigestellt am eigenen Promotionsprojekt $\mathrm{zu}$ arbeiten, entpuppt sich auf lange Sicht als nachteilig hinsichtlich fachlicher Einbindung, dem Zugang zu Netzwerken und alltagsnahen Kontakten im Wissenschaftsbetrieb (Bochow \& Joas, 1987; Czock \& Wildt, 1985; Holzbecher et al., 2002; Koch, 1995; Kort-Krieger, 1995; Mesletzky et al, 1995; Pfister, 1986; Röbbecke, 2004). Während Promotionsstipendiatinnen größtenteils nach Abschluss der Promotion keine wissenschaftliche Laufbahn weiterführen konnten (Egger, 1996; Kort-Krieger, 1995; Mesletzky et al., 1995), waren später erfolgreich Professorinnen während ihrer Qualifikationsphase überwiegend an einer Universität beschäftigt (Krimmer \& Zimmer, 2003).

Aufschlussreich sind auch Ergebnisse, die sich auf das Erleben von Bestätigung während der Promotionsphase und die Integration in die Scientific Community beziehen: Die Doktorandinnen fühlen sich weniger ermutigt und unterstützt, auch im Vergleich zu männlichen Doktoranden mit gleichem Status (Allmendinger, 2005; Geenen, 2000; Holzbecher et al., 2002; Pfister, 1986; Vogel \& Hinz, 2004). Selbst erfolgreiche Nachwuchswissenschaftlerinnen erleben sich nur zum Teil gut in die Scientific Community ihres Faches integriert (Geenen, 2000; Leemann, 2002; Lind, 2004b; Lind \& Löther, 2006; Vogel \& Hinz, 2004). Gerade diejenigen Frauen, die wenig Anerkennung und Ermutigung während der Promotion erfahren, sind für den Abbruch der wissenschaftlichen Laufbahn vulnerabel (Allmendinger et al, 1999; Eggers, 1996; Geenen, 2000; Holzbecher et al, 2002; Kracke \& Englich, 1996; v. Stebut, 2003; Vogel \& Hinz, 2004). Unklar ist jedoch, über welche konkreten Interaktionsprozesse sich die 
schrittweise Desintegration vollzieht und in welchem Maße unbewusste und bewusste Motivationslagen bei den Beteiligten eine Rolle spielen. Erfahrungsberichte von Wissenschaftlerinnen verweisen auf mehr oder weniger subtile Entwertungen, die über die Zuordnung bestimmter Aufgaben durch Vorgesetzte, den Ausschluss aus informellen Kreisen bis hin zur Nicht-Beachtung von Gesprächsbeiträgen reichen (Geenen, 2000; Krais \& Krumpeter, 1997; vgl. auch Allmendinger et al., Buchholz, 2004; 2001 Confrey, 2000; Enders \& Teichler, 1995; Gunter \& Stambach, 2005; Kiegelmann, 2000; Krimmer \& Zimmer, 2003; Malone et al, 2005; Matthies et al., 2001; Morrison et al, 2005; National Science Foundation, 2003; Seaman et al, 2001; zusammenfassend Lind, 2004a). Gleichzeitig stellt sich die Frage, ob bestimmte Interaktionen im Kontext der männlich dominierten Strukturen von den Wissenschaftlerinnen in anderer Weise interpretiert werden als von ihren männlichen Kollegen. Einige Autorinnen weisen darauf hin, dass es für Wissenschaftlerinnen einer besonderen Bestätigungskultur bedürfe, damit bestimmte soziale Interaktionen tatsächlich als bestätigend wahrgenommen werden (Allmendinger, 2005; Holzbecher et al., 2002; Lind, 2004b; Pfister; 1986).

Die vereinzelt vorliegenden Ergebnisse zur Situation von Habilitandinnen korrespondieren mit der Befundlage zum Promotionsprozess: Auch Habilitandinnen haben einen niedrigeren Stellenstatus als Habilitanden (Wissenschaftsrat, 1998) und fühlen sich oft wenig integriert und unterstützt (Kiegelmann; 2000; vgl. Lind, 2004b). Bemerkenswert und im Widerspruch zu verbreiteten Alltagsüberzeugungen ist, dass trotz vorherrschender eher traditioneller Geschlechtsrollenverteilung in den Partnerschaften beim Vorhandensein von Kindern sich die Produktivität der Mütter nicht von denen der kinderlosen Wissenschaftlerinnen unterscheidet (Kiegelmann, 2000; Lind, 2004b; vgl. dazu Leemann, 2002; Lind, 2004c). ${ }^{11}$

Auf der Ebene der Hochschulstrukturen wird vor allem die spezifische Form der Nachwuchsrekrutierung als ursächlich für den Frauenanteil in der Wissenschaft benannt: Im bundesdeutschen Hochschulsystem existiert kein formalisierter Zugang zu einem Promotionsstudium beispielsweise über zentrale Entscheidungsstrukturen oder Testverfahren, was sich - wie der Vergleich mit anderen Ländern zeigt - ungünstig auf den Frauenanteil in der Wissenschaft auswirkt (Costas, 2003). Durch fehlende formale Vorgaben gewinnen informelle Strukturen, Netzwerke und Entscheidungsmuster eine höhere Bedeutung. Gleichzeitig begünstigen informelle Strukturen eher diejenige Personengruppe, die in einer Organisation bereits die Mehrheit bildet, wie organisationssoziologische Ansätze herausgearbeitet haben (Krais \& Krumpeter, 1997; Matthies et al. 2001; Perkins, 1973). Der wenig formalisierte Zugang zu Qualifikationsstellen wird in den letzten Jahren zunehmend kritisiert (vgl. Wissenschaftsrat, 1998) und es mehren sich quantifizierbare Hinweise, dass Frauen von Ausschreibungsverfahren vergleichsweise stärker profitieren als von informellen Verfahren (CEWS, 2006; Costas, 2003). 
Aufgrund der eher informellen Stellenvergabepraxis kommt den individuellen Förderbeziehungen eine hohe Bedeutung zu (Allmendinger, 2005; Krimmer \& Zimmer, 2003; Wissenschaftsrat, 1998). Einerseits bietet eine individuelle Förderbeziehung den Zugang zu einem Promotionsthema, andererseits findet wissenschaftliche Anerkennung zu einem frühen Zeitpunkt der Laufbahn fast ausschließlich innerhalb dieser Förderbeziehung statt (Allmendinger, 2005; Krimmer \& Zimmer, 2003). Die große Bedeutung der individuellen Förderbeziehung gerade zu Beginn einer wissenschaftlichen Karriere bei gleichzeitigem Mangel an fördernden weiblichen Modellen wird in jüngerer Zeit als bedeutsame Barriere für Frauen benannt. Die Nachwuchsrekrutierung vollzieht sich faktisch vor allem nach Prozessen der homosozialen Kooptation (Allmendinger, 2005; Costas, 2003). Spätere Professoren und Professorinnen berichten, dass überwiegend gleichgeschlechtliche Förderbeziehungen während der wissenschaftlichen Laufbahn eine wichtige Rolle gespielt hätten (Krimmer \& Zimmer, 2003). Aufgrund des Frauenanteils an Professorinnen sind somit Nachwuchswissenschaftlerinnen strukturell benachteiligt. Bei gleich bleibender Rekrutierungspraxis wiederholen sich die selbstreproduzierenden Prozesse und machen dynamische Veränderungen unwahrscheinlicher.

\section{Disziplinäre Unterschiede}

\section{Ergebnisse der fächerspezifischen Datenanalyse}

Bisher wurden Prozesse der Marginalisierung von Frauen in der Wissenschaft vorwiegend übergreifend für alle Fächer untersucht. Im vorliegenden Datenmaterial zeigen sich aber deutliche Unterschiede zwischen den Fächergruppen: Besonders offensichtlich treten diese im Vergleich der Sprach- und Kulturwissenschaften mit den Ingenieurwissenschaften auf. Fächergruppen mit Frauenanteilen von mehr als 60 Prozent bei den Studienanfängern zeigen bei den Berufungen die höchsten Verluste (35 - 40 Prozentpunkte). Von den Studienanfängern in den Sprach- und Kulturwissenschaften im Jahr 1986 waren 71 Prozent Frauen. Ihr Anteil an den Neuberufungen in dieser Fächergruppe war 2003-2005 mit 40 Prozent zwar vergleichsweise hoch; im Vergleich zum vorhandenen Potenzial an Studienanfängerinnen und Absolventinnen ergibt sich jedoch ein Rückgang um 31 Prozentpunkte. Entscheidend ist dabei der Übergang in die Promotion: Die Promotionsintensität der Absolventen der Jahre 1991-93 in den Sprach- und Kulturwissenschaften liegt bei 8,3 Prozent (in Bezug auf eine Promotion vier Jahre später), die der Absolventinnen bei 5,2 Prozent. Im Übergang zur Habilitation sinkt der Frauenanteil dann lediglich um rund 7 Prozentpunkte und der Frauenanteil an den Erstberufungen liegt fast auf dem Niveau der Promotionen. Die wenigen habilitierten Frauen hatten damit einen relativ guten Zugang zu Professuren. Interessant wäre hier eine Analyse der einzelnen Fächer; für Berufungen liegen jedoch keine nach Fächern differenzierten Daten 
vor. $\mathrm{Zu}$ vermuten ist, dass in Fächern mit besonders hohem Frauenanteil - wie Germanistik oder Romanistik - im Vergleich zu Fächern wie Geschichte oder Philosophie mit einem Studentinnenanteil unter 50 Prozent weniger Frauen ihre wissenschaftliche Qualifikation nach dem ersten Abschluss fortsetzen.

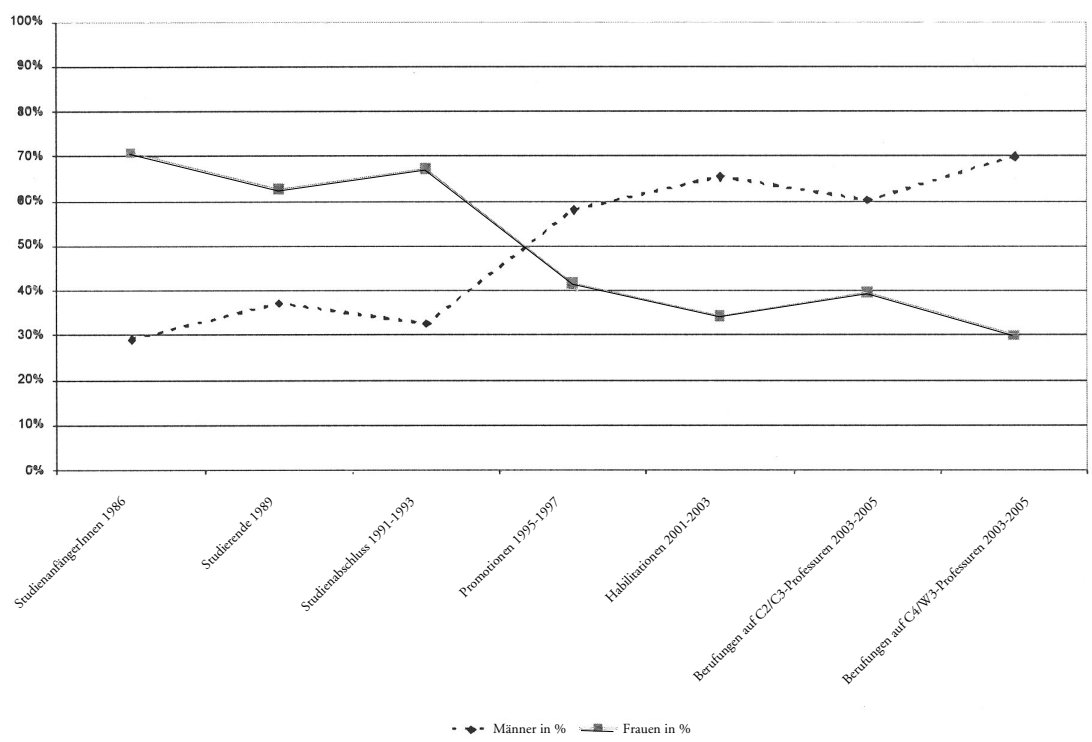

Abbildung 3: Frauen- und Männeranteile in den Sprach-und Kulturwissenschaften

Ähnliche große Differenzen zwischen den Frauenanteilen an den Studienanfängern und an den Erstberufungen zeigen sich in der Human- und Veterinärmedizin sowie den Agrar-, Ernährungs- und Forstwissenschaften. Die Veterinärmedizin ist mit einem Frauenanteil von 62 Prozent ähnlich stark weiblich dominiert wie die Sprach- und Kulturwissenschaften. Bis zu den Erstberufungen sinkt der Frauenanteil um 35 Prozentpunkte auf 27 Prozent und hat sich damit mehr als halbiert. In der Humanmedizin lag der Frauenanteil Mitte der 1980er Jahre bei 40 Prozent. $^{12}$ Jedoch gingen nur 11 Prozent der Erstberufungen in dieser Fächergruppe 2003-2005 an Frauen, ein Rückgang um 31 Prozentpunkte gegenüber den Studienanfängerinnen des Jahres 1986. Entsprechend der Besonderheit der medizinischen Fächer erfolgt der Rückgang des Frauenanteils erst mit der Habilitation. Sowohl in der Humanmedizin als auch in der Veterinärmedizin geht der Frauenanteil an den Habilitationen im Vergleich zur Promotion um rund 25 Prozentpunkte zurück. Auffällig ist weiter, dass nur in den medizinischen Fächern der Frauenanteil an den Erstberufungen niedriger ist als an den Habilitationen; habilitierte Medizinerinnen haben also schlechtere Chancen auf eine Berufung als Mediziner. ${ }^{13}$ 


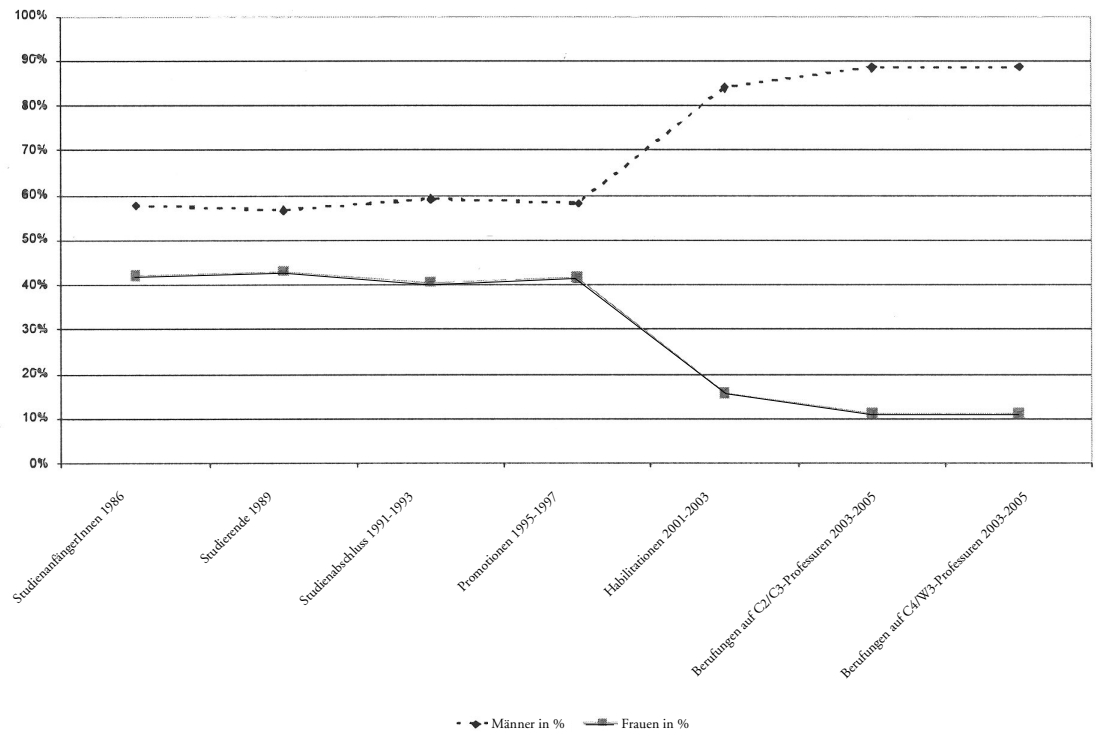

Abbildung 4: Frauen- und Männeranteile in der Humanmedizin

Interessante Ergebnisse zeigen sich bei der Fächergruppe der Rechts-, Wirtschafts- und Sozialwissenschaften. Bei einem Frauenanteil von fast 50 Prozent an den Studienanfängern 1986 sind ein deutlicher Rückgang bei den Promotionen (25 Prozentpunkte und damit Halbierung des Frauenanteils) und ein geringerer Rückgang bei den Habilitationen (8 Prozentpunkte) festzustellen. Auffällig ist nun, dass der Frauenanteil an den Berufungen auf C2-/C3- und W2-Professuren um Prozentpunkte höher liegt als der Anteil an den Habilitationen und sogar noch um 10 Prozentpunkte höher als der Frauenanteil an den Promotionen ist. Eine Erklärung hierfür mag in dem Umstand liegen, dass fast drei Viertel aller Berufungen in dieser Fächergruppe an Fachhochschulen erfolgt und dort eine Habilitation nicht Voraussetzung für die Professur ist. Zudem liegt der Frauenanteil an Fachhochschulberufungen in dieser Fächergruppe mit 34,4 Prozent höher als an Universitäten (27,9 Prozent). Eine aktive Berufungspolitik der Fachhochschulen zugunsten von Frauen gerade in der Fächergruppe der Rechts-, Wirtschafts- und Sozialwissenschaften könnte den im Vergleich mit den Promotionen hohen Frauenanteil an Berufungen begründen. Aufgrund fehlender nach Fächern differenzierter Daten für Neuberufungen ist es leider nicht möglich zu klären, ob es Unterschiede zwischen den Sozialwissenschaften, der Rechtswissenschaft und den Wirtschaftswissenschaften gibt. ${ }^{14}$ 


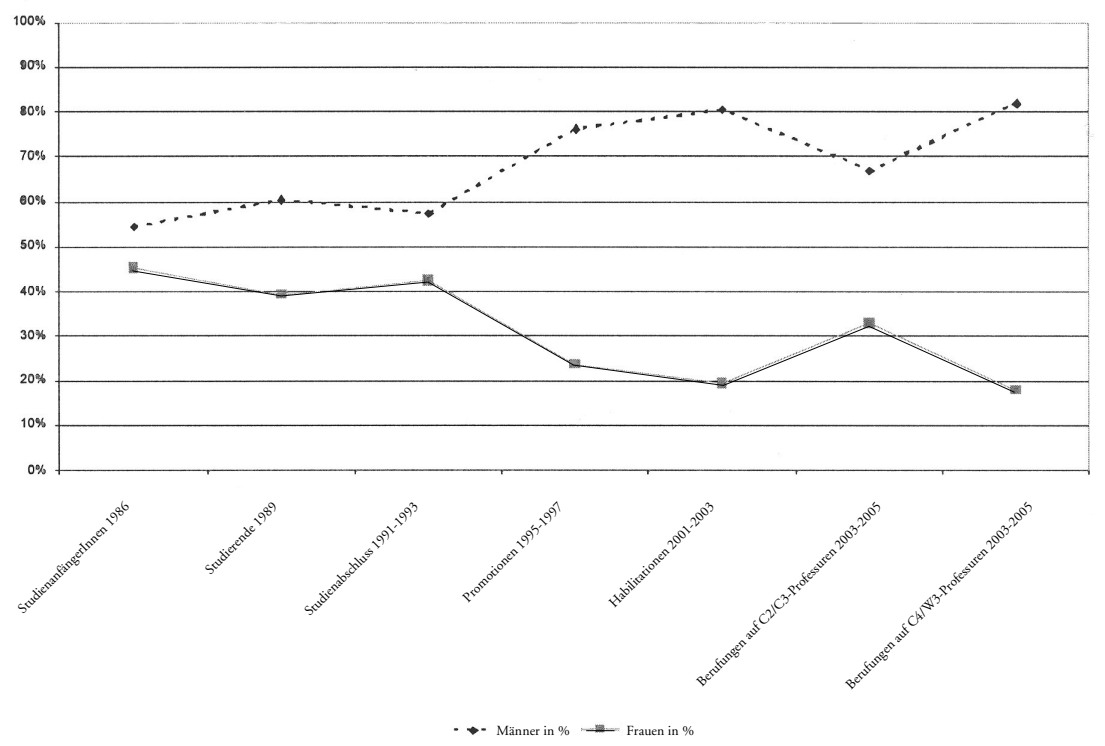

Abbildung 5: Frauen- und Männeranteile in den Rechts-, Wirtschafts- und Sozialwissenschaften

Anders als alle anderen Fächergruppen stellt sich die Entwicklung der Frauenund Männeranteile im Qualifikationsverlauf für die Ingenieurwissenschaften nicht als Scherendiagramm dar: Der Frauenanteil von 11,2 Prozent an den Erstberufungen 2003-2005 war ebenso hoch wie der Frauenanteil an Studienanfängern 1986 (11,7 Prozent). Der Selektionsprozess findet in dieser Fächergruppe, die den niedrigsten Studentinnenanteil aller Fächergruppen aufweist, bereits im Zugang zum Studium statt. Für die Ingenieurwissenschaften ist allerdings zu befürchten, dass auch hier mit steigenden Studentinnenanteilen der Frauenanteil in den höheren Qualifikationsstufen nicht entsprechend wächst. So waren 1995 19,7 Prozent der Studienanfänger Frauen. Während sich der Frauenanteil an den Abschlüssen bis 2001 ebenfalls auf 19,8 Prozent steigerte, lag er 2005 bei den Promotionen mit 13,6 Prozent deutlich unter dieser Steigerungsrate.

In der Fächergruppe Mathematik und Naturwissenschaften wiederum fällt der Frauenanteil an den Studienanfängern von 35 Prozent auf 18 Prozent bei den Berufungen. Allerdings ist der Rückgang nicht so stark wie in den Fächern mit sehr hohem Frauenanteil. Zu vermuten sind Unterschiede innerhalb dieser Fächergruppen zwischen Fächern mit einem hohen Frauenanteil wie Biologie und Pharmazie und von Männern dominierten Fächern wie Physik oder Informatik. So lag der Frauenanteil an den Habilitationen in letzteren Fächern 2001-2003 lediglich vier bis fünf Prozentpunkte niedriger als der Anfängerinnenanteil 1986 (13 Prozent für Physik und 16 Prozent für Informatik), während in Pharmazie ein Rück- 
gang um 46 Prozentpunkte, in Biologie um 33 Prozentpunkte zu verzeichnen ist.
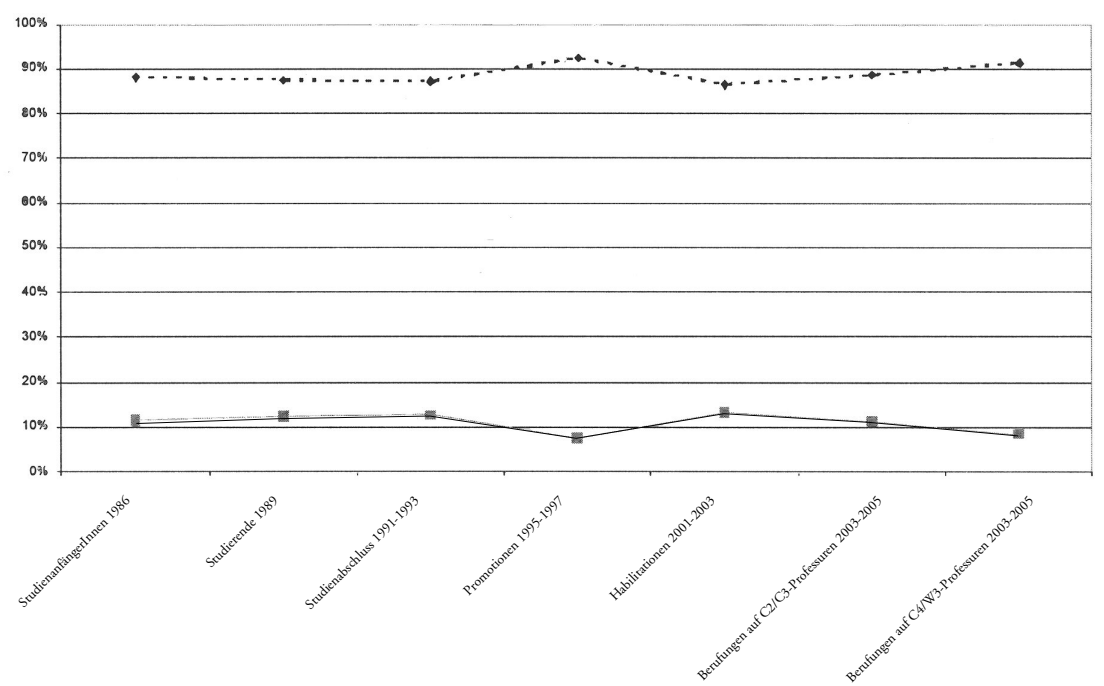

Abbildung 6: Frauen- und Männeranteile in den Ingenieurwissenschaften

\section{Erklärungsansätze zu disziplinären Unterschieden}

Die deutlichen disziplinären Unterschiede fanden bislang nur wenig Resonanz von Seiten der Wissenschaftsforschung. ${ }^{15}$ Entsprechende Erkenntnisse entstammen den wenigen Studien zu Fachkultur und Geschlecht (Engler, 1999; Kirschbaum et al., 2005; Heintz, Merz \& Schumacher, 2004; Holzbecher et al, 2002; Mischau et al., 2004) bzw. Arbeiten, die neben anderen Themenschwerpunkten auch Hinweise auf fächerspezifische Wirkstrukturen beinhalten (Geenen, 1994; Geenen 2000; Leeman 2002; Röbbecke \& Simon; 2001 Stegmann, 2005; Vogel \& Hinz, 2004).

Die von verschiedenen Autoren formulierten Thesen zur differentiellen Wirkung von Fachkulturen beziehen sich auf die unterschiedlichen epistemischen Räume, die - so die Annahme - mit unterschiedlichen Spielräumen für die Darstellung von Geschlecht verbunden sind (Heintz et al., 2004). Zunehmend Bedeutung gewinnt die These vom Formalisierungsgrad einer Disziplin: Mit stärker formalisierter Studienstruktur sei eine Trennung von Leistung und Person eher möglich als in Fächern mit geringem Formalisierungsgrad (Geenen, 1994; Heintz et al., 2004), da die wenig formalisierten Leistungskriterien mehr Raum für geschlechtsspezifische Zuschreibungen ließen, die sich in einer zurückhaltenden Förderung von Frauen niederschlügen (Kirschbaum et al., 2005; siehe auch Leemann, 2002). Eine weitere These bezieht sich auf die Wir- 
kung vorhandener Drittmittel: Kirschbaum und Kolleginnen vermuten, dass in Disziplinen mit hohem Drittmittelaufkommen Frauen mehr Chancen haben, in ihrer Promotion gefördert zu werden als in Fächern mit geringem Drittmittelaufkommen. Für die Mehrzahl der überwiegend von Frauen studierten Fächer sei jedoch ein vergleichsweise geringes Drittmittelaufkommen kennzeichnend (Kirschbaum et al., 2005, S. 177ff).

Die Ergebnisse der einzelnen Studien stellen sich in der Zusammenschau als teilweise widersprüchlich dar, so zum Beispiel hinsichtlich der Größe der Disziplinen. Nach Geenen (1994) fühlen sich Studentinnen in großen Fächern weniger diskriminiert, da die Größe des Faches durch standardisierte Leistungsnachweise egalisierend wirke. Die Nachwuchsrekrutierung findet laut Geenen (1994) in den großen Fächern zu einem späteren Zeitpunkt im Studium statt und biete damit zunächst mehr Offenheit und geringe Festlegung zu Beginn des Studiums (Geenen, 1994, S. 64). Leemann (2002) konnte dagegen für die Schweiz keine Unterschiede zwischen großen und kleinen Fächern hinsichtlich der Einbindung von Frauen feststellen. Deutlichere Belege gibt es für die Bedeutung des Standardisierungsgrades, wonach Frauen von standardisierten Vorgaben stärker profitieren, wie z.B. in den Natur- und Ingenieurwissenschaften (Heintz et al., 2004, S. 65; vgl. Costas, 2003).

Fachkulturspezifische Ausdrucks- und Darstellungsformen in Bezug auf die Einbindung von Frauen sind erst in jüngster Zeit Gegenstand der Forschung. Insgesamt liegen aber bislang nur sehr wenige Studien zu einzelnen Disziplinen vor, so dass sich zum gegenwärtigen Zeitpunkt keine allgemeinen Aussagen ableiten lassen. Wichtig erscheint in diesem Zusammenhang jedoch, dass insbesondere in Disziplinen mit hohem gesellschaftlichen Ansehen die informellen Codes zu einer starken Geschlechterselektion führen (Stegmann, 2005, S. 26). Die Unterschiede zwischen den einzelnen Fachkulturen zeigen sich in älteren Universitäten stärker als in jüngeren Hochschulen (Stegmann, 2005, S. 238ff.).

Von besonderem Interesse ist die Frage, warum sich in den sogenannten feminisierten Fächern ${ }^{16}$ der Minderheitenstatus der Männer in prozentual starker Beteiligung am wissenschaftlichen Qualifikationsprozess niederschlägt, bei umgekehrter Geschlechterkonstellation sich Frauen jedoch nicht überproportional wissenschaftlich weiterqualifizieren. Holzbecher et al. (2002) nehmen dazu an, dass aufgrund der geringen Reputation der Fächer mit hohem Frauenanteil die jungen Männer bereits zu einem frühen Zeitpunkt ein besonderes Qualifikationsbedürfnis entwickeln, was von Seiten der Hochschullehrenden in entsprechender Weise beantwortet wird. Am Beispiel der Botanik zeigen Heintz et al. (2004, S. 203 ff) auf, wie die geringe Reputation des Faches als typisches Frauenfach, die Geschlechterkonstellation innerhalb des Faches und die Berufsaussichten in Wechselbeziehungen miteinander stehen und zu Nachteilen für potentielle Nachwuchswissenschaftlerinnen führen. Ebenfalls existieren Hinweise, dass Doktorandinnen in den weiblich dominierten Fächern stärker in die 
Lehre eingebunden sind als Naturwissenschaftlerinnen und daher weniger Zeit für die eigene Qualifikation aufbringen können (Kirschbaum et al., 2005; Heintz et al., 2004, S. 66).

Es steht zu vermuten, dass der Verlust an Reputation, der mit einem steigenden Frauenanteil in einer Disziplin einhergeht, nicht nur bei männlichen Studierenden zu einem besonderen Abgrenzungs- und Profilierungsbedürfnis führt, sondern auch von der Fachelite in spezifischer Weise beantwortet wird. So sind intensivierte, am Prinzip der homosozialen Kooptation orientierte Selektionsmechanismen durch die Fachelite als wahrscheinlich anzunehmen, wie sie auch in anderen Bereichen der Elitebildung bereits beschrieben wurde (Bourdieu \& Passeron 1971; Hartmann 2005). Auch zu diesem Aspekt von Fachkulturen steht eine systematische Auseinandersetzung noch aus.

Einen interessanten Ansatzpunkt bietet die Frage der Vorselektion zu Beginn des Studiums und den damit verbundenen Prozessen der Auseinandersetzung. So ist die Entscheidung einer Frau für das Fach Elektrotechnik mit anderen Erwartungen, Befürchtungen und Bewältigungsprozessen verbunden als die Entscheidung einer Frau für ein Fach mit einem hohen Studentinnenanteil. Junge Männer, die ein weiblich dominiertes Studienfach wählen, sehen sich mit latenten Abwertungen durch das soziale Umfeld konfrontiert. Insofern gehen einer scheinbar ähnlichen Situation, nämlich dem Minderheitenstatus in einer Disziplin, je nach Geschlechterkonstellation andere Entscheidungs- und Auseinandersetzungsformen voraus und führen mit hoher Wahrscheinlichkeit zu unterschiedlichen Bewältigungsmustern, die beispielsweise zu einem besonderen Profilierungsbedürfnis von Männern führen können (vgl. dazu auch Heintz et al., 2004). Insgesamt gibt es jedoch zu Selektionsprozessen und deren Bedingungsfaktoren im Vorfeld des Studiums erst wenige Erkenntnisse (Ostendorf, 2005).

Die Betrachtung der Berufschancen außerhalb der Wissenschaft ermöglicht einen weiteren ergänzenden Blick auf disziplinäre Unterschiede. So steht geschlechtsspezifisches Promotionsverhalten auch in Bezug zu der Verwertbarkeit der Promotion außerhalb der Wissenschaft. In Disziplinen, in denen eine Promotion keine quasi regelhafte Vorbedingung für den Berufseinstieg darstellt, neigen Frauen stärker dazu, sich auf das eigentliche Berufsbild zu konzentrieren und nicht bereits zu einem frühen Zeitpunkt mit Blick auf die Erreichung einer statushohen Position eine Promotion anzustreben (Kirschbaum et al., 2005; Spieß \& Schute, 1999). Andererseits kann die Wissenschaft für Frauen eine vergleichsweise attraktive Alternative darstellen, beispielsweise wenn das Berufsfeld außerhalb der Wissenschaft als problematisch eingeschätzt wird. Schreyer (1999) verwies darauf, dass Technik- und Ingenieurwissenschaftlerinnen nur geringe Berufsaussichten auf dem außeruniversitären Arbeitsmarkt für sich als Frauen antizipieren. ${ }^{17}$ Die relativ hohe Promotionsrate von Frauen in den Ingenieurwissenschaften kann vermutlich nicht unabhängig von diesen außeruniversitären Bedingungen gesehen werden. 
Internationale Vergleiche zu Frauen- und Männeranteilen in verschiedenen Disziplinen zeigen eindrucksvoll, wie stark die geschlechtsspezifische Fächerwahl und die Einbindung von Frauen in den Qualifikationsprozess durch soziokulturelle Zuschreibungen moderiert sind. ${ }^{18}$ Während im deutschsprachigen Raum beispielsweise die Physik besonders männlich konnotiert ist, gilt das Fach in manchen osteuropäischen Ländern als eher feminisiertes Fach. ${ }^{19}$ Grundlegend für diese Unterschiede sind laut Stadler (2005) unter anderem Vorstellungen vom Schwierigkeitsgrad eines Faches. Während die Physik hierzulande als besonders anspruchsvoll und prestigeträchtig gilt, hat diese Disziplin in Osteuropa kein Image als schwieriges Fach. In Indien gilt Physik sogar als schöngeistiges Frauenfach, dass von Männern so gut wie nicht gewählt wird (Ratzer et al., 2006). Noch gibt es jedoch keine Studien, die den Zuschreibungsprozessen als männliche oder weibliche Disziplin nachgehen. Gut zu beobachten ist jedoch am Beispiel der ehemaligen DDR, wie schnell sich unter neuen gesellschaftlichen Bedingungen fachspezifische Zuschreibungen ändern (Stadler, 2005).

Die dargestellten Ergebnisse vermitteln einen Eindruck von den komplexen Verschränkungen zwischen Fachkultur und Geschlecht, die jeweils starken gesellschaftshistorischen und kulturellen Prägungen unterliegen und in ihrer Konsequenz zu einer unterschiedlichen Durchlässigkeit für Frauen führen. Der Kenntnisstand zu den einzelnen Bedingungsfaktoren ist jedoch noch sehr unvollständig, wie auch Heintz und Kolleginnen resümieren: «Im Gegensatz jedoch zur Geschlechterforschung, wo die theoretische und methodische Reflexion der Grundkategorie Geschlecht weit fortgeschritten ist, hat die Wissenschaftsforschung die Frage der disziplinären Unterschiede noch kaum systematisch behandelt, weder empirisch, noch methodisch oder theoretisch» (Heintz et al., 2004, S. 186).

\section{Fazit}

Insgesamt macht die Analyse deutlich, dass in allen Fächergruppen außer in den Ingenieurwissenschaften mit Studienanfängerinnen und Absolventinnen, die ihr Studium in den 1980er Jahren absolvierten, ein Pool an potenziellen Wissenschaftlerinnen zur Verfügung stand, der jedoch nicht genutzt wurde. Die Unterrepräsentanz von Wissenschaftlerinnen in Führungspositionen kann in fast allen Fächergruppen also nicht auf das fehlende weibliche Potenzials in der jeweils vorhergehenden Qualifikationsstufe zurückgeführt werden. Insbesondere wurde auch deutlich, dass der Frauenanteil in weiblich dominierten Fächergruppen wie den Sprach- und Kulturwissenschaften überproportional sinkt und eine Förderung von Wissenschaftlerinnen in diesen Fächern im Vergleich zum vorhandenen Potenzial schlechter gelingt. Dieser Verlust des weiblichen Potenzial für die Wissenschaft lässt sich noch an einer andern Kennziffer deutlich machen, der Chance von Absolventen eine Professur zu erlangen: Über alle Fächer hinweg 
hatten Absolventen der Jahrgänge 1991-93 eine fast dreimal so hohe Chance auf eine Professur wie ihre Kommilitoninnen: 1,2 Prozent der Absolventen gegenüber 0,4 Prozent der Absolventinnen wurden auf eine Professur berufen.

Darüber hinaus werden fächerspezifische Prozesse und Kulturen in der Qualifikationsphase und hinsichtlich der Ausschlussmechanismen gegenüber Frauen deutlich. Forschungsdesiderate bestehen hinsichtlich der geschlechtsspezifischen Zuschreibungsprozesse einzelner Disziplinen, der spezifischen Wirkung von Fachkulturen unter dem Geschlechteraspekt und den ursächlichen Faktoren für die geschlechtspezifischen Aufstiegsbedingungen in den überwiegend von Frauen studierten Fächern. Auch die Wechselwirkungen von individuellen Faktoren, den Fachkulturen und Bedingungen auf dem außeruniversitären Arbeitsmarkt verdienen verstärkt Aufmerksamkeit, ebenso wie die sozio-kulturellen Einflüsse zur Erklärung des geschlechtsspezifischen Studienwahlverhaltens und nationaler Unterschiede. Bislang wenig beachtet wurden Prozesse der Vorselektion zu Beginn eines Studiums. Gerade für karriererelevantes Verhalten während der akademischen Ausbildung erscheint dies als lohnendes und bislang weitgehend unbeachtetes Feld. Es bedarf einer verstärken Analyse und Auseinandersetzung mit einzelnen Fachkulturen und disziplinären Unterschieden, um passgenaue gleichstellungspolitische Maßnahmen zu entwickeln.

\section{Anmerkungen}

1 Destatis, Bildung und Kultur - Studierende insgesamt, URL: http://www.destatis.de/basis/d/biwiku/hochtab2.php, aufgenommen am 25.10.2006. Pressemitteilung vom 6. Juli 2006, URL: http://www.destatis.de/presse/deutsch/pm2006/p2700071.htm. Universitätsbericht 2005, Bd. 1: 131 (die österreichischen Daten beziehen sich auf Vollzeitäquivalente). bm:bwk: Statistiken zum österreichischen Universitätswesen, Geschlechteranteile (in Prozent) der Studierenden, Erstzugelassenen und Neuzugelassenen an Universitäten der Wissenschaften und Universitäten der Künste, URL: http://www.bmbwk.gv.at/medienpool/13145/geschlechteranteile05.pdf. Statistik Schweiz, Personalmerkmale Hochschulen: Frauenanteile nach Personalkategorie 2001-2005,

URL: http://www.bfs.admin.ch/bfs/portal/de/index/themen/15/06/key/ind12.indicator.12309.html?open=143\#143, aufgenommen am 25.10.2006.

2 Angaben für Österreich für 2002. International vergleichbar ist nicht die Kategorie «Professuren». Stattdessen wurde eine Einteilung der Positionen an Hochschulen oberhalb der Promotion vorgenommen mit Grade A definiert als «The single highest grade/post at which research is normally conducted» (European Commission, 2006, S. 56). Für Deutschland werden dieser Kategorie C4/W3-Professuren zugeordnet. Die Schweiz hatte 2004 in dieser Kategorie einen Frauenanteil von 16,5 Prozent.

3 Erstmalig veröffentlich in: BLK 2005.

4 Vgl. dazu auch die aktuelle Studie von Janson, Schomburg und Teichler (2006). Nach ihnen variiert die Dauer zwischen Promotion und Habilitation zwischen 6 und 9 Jahren. Sie gehen von einer durchschnittlichen Dauer von fast 8 Jahren aus, Habilitationen in der Human- und Veterinärmedizin eingeschlossen.

5 Vgl. auch Janson et al. (2006), die einerseits von einer Zeitspanne von gut 20 Jahren ausgehen, andererseits annehmen, dass diejenigen, «die schließlich eine Professur erreichen, vor den durchschnittlichen Werten die vorangehende Qualifikation erreichen» (103) und die Qualifikationsphase eher auf etwas über 15 Jahre einschätzen. 
6 Die BLK veröffentlicht seit 1997 jährlich geschlechterspezifische Daten zu Hochschulen und Forschungseinrichtungen, darunter Daten zum Berufungsgeschehen. Vgl. als aktuelle Veröffentlichung BLK 2006.

72004 regten die europäischen Forschungs- und Bildungsminister und -ministerinnen die Erhebung von Rekrutierungsdaten an. Conclusions of the Competitiveness Council (Internal market, Industry and Research) - 18 April 2005

(URL: http://www.eu2005.lu/en/actualites/conseil/2005/04/18compet/84579.pdf).

8 Eine Kurzfassung ausgewählter Ergebnisse der nachfolgend dargestellten statistischen Analysen erschien in Forschung \& Lehre 11/2006, S. 634.

9 Vgl. CEWS-Statistikportal, Entwicklung des Studentinnenanteils in Deutschland seit 1908, URL: http://www.cews.org/statistik/hochschulen.php?aid=20\&cid=16.

10 Für eine ausführliche Darstellung der Entwicklung des Forschungsstranges zur Marginalisierung von Frauen in der Wissenschaft siehe Lind, 2004a.

11 Leemann (2002) konnte zeigen, dass ein vorhandenes Kleinkind keine statistisch relevante Bedeutung für den Übergang in eine wissenschaftliche Laufbahn hat. Weiter berichtet Leemann, dass sich negative Effekte eines Kleinkindes wenn überhaupt, dann eher bei männlichen Wissenschaftlern zeigen (Leemann, 2002, S. 166).

12 Aktuell sind fast zwei Drittel der Studienanfänger in Human- und Zahnmedizin Frauen

13 Zur besonderen Situation von Frauen in der Medizin vgl. auch BLK 2004.

14 Da der Frauenanteil an den Professuren in den Sozialwissenschaften 2003 mit 23 Prozent doppelt so hoch war wie in den Rechts- und in den Wirtschaftswissenschaften ist zu vermuten, dass es auch Unterschiede bei den Berufungen gibt.

15 Die Anzahl der derzeit noch laufenden Forschungsprojekte zu disziplinären Unterschieden sprechen dafür, dass sich das Forschungsinteresse zunehmend auf die disziplinären Unterschiede konzentriert.

16 Der Begriff ,feminisiertes Fach' wird verwendet zur Bezeichnung von Disziplinen mit einem Frauenanteil von über 60 Prozent. Problematisch ist der Begriff insofern, als neben der neutralen Bezeichnung eines hohen Frauenanteils das Attribut feminisiert auch assoziativ mit bestimmten Attributen von Weiblichkeit verbunden ist. In der Vergangenheit ging die ,Feminisierung' einzelner Disziplinen und Professionen häufig mit deren Statusverlust einher (vgl. z.B. Wetterer 1994). Insofern bedarf der weitgehend im Konsens verwendete Begriff der so genannten feminisierten Fächer einer kritischen Reflexion bzgl. des darin enthaltenden latent diskreditierenden Potentials. $\mathrm{Zu}$ analysieren wäre dabei auch die Verwendung und der Gebrauch des Begriffs ,maskulinisiertes Fach'.

17 Gleichzeitig arbeitet ein hoher Anteil von Absolventinnen der technik- und ingenieurwissenschaftlichen Fächer nicht in ihrem eigentlichen Berufsfeld; siehe Plicht \& Schreyer, 2002; Haffner, Könekamp und Krais, 2006.

18 So wurden im Jahr 2003 in Litauen rund 44\% der Promotionen in Ingenieurwissenschaften von Frauen abgelegt; in Polen stammten 2003 50\% aller Promotionen der Fächergruppe ,Science, Mathematics and Computer' von Frauen. Quelle: Eurostat. Vgl. dazu auch European Commission, 2006.

19 Vgl. dazu European Commission, 2006.

\section{Literatur}

Achatz, J.,Allmendinger, J., Hinz, T. \& Priel, P. (2000). Growth to Limits? Zur Integration von Frauen in Organisationen im zeitlichen Verlauf? In H. Schwengel (Hrsg.), Grenzenlose Gesellschaft? 29. Kongress der Deutschen Gesellschaft für Soziologie. Pfaffenweiler: Centaurus.

Allmendinger, J., Fuchs, S. \& v. Stebut, J. (1999). Drehtüre oder Paternoster? Zur Frage der Verzinsung der Integration in wissenschaftliche Organisationen im Verlauf beruflicher 
Werdegänge von Wissenschaftlerinnen und Wissenschaftlern. In C. Honegger, S. Hradil \& F. Traxler (Hrsg.), Grenzenlose Gesellschaft? Verhandlungen des 29. Kongresses der Deutschen Gesellschaft für Soziologie, des 16. Kongresses der Österreichischen Gesellschaft für Soziologie, des 11. Kongresses der Schweizerischen Gesellschaft für Soziologie in Freiburg i.Br. 1998 (S. 96-107), Teil 2. Opladen: Leske und Budrich.

Allmendinger, J., Fuchs, S., v. Stebut, J. \& Wimbauer, C. (2001). Contested Terrain: Women in German Research Organizations. In V. W. Marshall et. al. (Eds.), Restructuring Work and the Life Course (p. 107-122). Toronto/Buffalo/London: University of Toronto Press. Allmendinger, J. (2005). Fördern und Fordern - was bringen Gleichstellungsmaßnahmen in Forschungseinrichtungen? Empirische Ergebnisse. In A. Spellerberg (Hrsg.), Die Hälfte des Hörsaals: Frauen in Hochschule, Wissenschaft und Technik (S. 51-74). Berlin: Edition Sigma

BLK (Bund-Länder-Kommisson für Bildungsplanung und Forschungsförderung) (2004). Frauen in der Medizin, Ausbildung und berufliche Situation von Medizinerinnen, Bericht der BLKvom 5. Juli 2004. (Materialien zur Bildungsplanung und Forschungsförderung, Heft 117). Bonn: BLK.

BLK (Bund-Länder-Kommisson für Bildungsplanung und Forschungsförderung) (2005). Frauen in Führungspositionen an Hochschulen und außerhochschulischen Forschungseinrichtungen, neunte Fortschreibung des Datenmaterials. (Materialien zur Bildungsplanung und Forschungsförderung, Heft 129) Bonn: BLK

BLK (Bund-Länder-Kommisson für Bildungsplanung und Forschungsförderung) (2006). Frauen in Führungspositionen an Hochschulen und außerhochschulischen Forschungseinrichtungen, zehnte Fortschreibung des Datenmaterials. (Materialien zur Bildungsplanung und Forschungsförderung, Heft 136) Bonn: BLK

Bochow, M. \& Joas, H. (1987). Wissenschaft und Karriere: Der berufliche Verbleib des akademischen Mittelbaus. Frankfurt/M.: Campus.

Bourdieu, P. \& Passeron, J.-C. (1971). Die Illusion der Chancengleichheit: Untersuchungen zur Soziologie des Bildungswesens am Beispiel Frankreichs. (Veröffentlichungen des Max-PlanckInstituts für Bildungsforschung). Stuttgart: Klett.

Buchholz, L. (2004). Wissenschaftskarrieren an österreichischen Universitäten. Erfahrungen und Einstellungen von Professorinnen und Professoren. In E. M. Appelt (Hrsg.), Karrierenschere. Geschlechtsverhältnisse im österreichischen Wissenschaftsbetrieb (S. 71-91). Wien: LIT Verlag.

CEWS (Kompetenzzentrum Frauen in Wissenschaft und Forschung) (2006). Juniorprofessuren in Nordrhein-Westfalen. Ein Vergleich der Qualifikationswege Juniorprofessur und C1-Assistentenstelle. (Bisher unveröffentlichter Bericht des Kompetenzzentrums Frauen in Wissenschaft und Forschung, Bonn).

Costas, I. (2003). Diskurse und gesellschaftliche Strukturen im Spannungsfeld von Geschlecht, Macht und Wissenschaft. Ein Erklärungsmodell für den Zugang von Frauen zu akademischen Karrieren im internationalen Vergleich. In I. Amodeo (Hrsg.), Frau Macht Wissenschaft. Wissenschaftlerinnen gestern und heute (S. 157-181). Königstein,Taunus: Helmer.

Czock, H. \& Wildt, J. (1985). Doktoranden. Eine von der Max-Traeger-Stiftung geförderte Untersuchung der Lage des wissenschaftlichen Nachwuchses. Freiburg i. Br.: Dreisam-Verlag

Eggers, S. (1996). Die Stipendiat(inn)enförderung als Wissenschaftler(innen)förderung: berufssoziologische Überlegungen zu einem ausgelagerten Schauplatz der Profession. In B. Kracke (Hrsg.), Arbeitsplatz Hochschule: Überlegungen und Befunde zur beruflichen Situation und Förderung des wissenschaftlichen Nachwuchses (S. 159-170). Heidelberg: Mattes

Enders, J. (1996). Die wissenschaftlichen Mitarbeiter: Ausbildung, Beschäftigung und Karriere der Nachwuchswissenschaftler und Mittelbauangehörigen an den Universitäten. Frankfurt/Main; New York: Campus-Verlag.

Enders, J. \& Teichler, U. (1995). Berufsbild der Lehrenden und Forschenden an Hochschulen. Ergebnisse einer Befragung des wissenschaftlichen Personals an Westdeutschen Hochschulen. 
Hrsg. vom Bundesministerium für Bildung, Wissenschaft, Forschung und Technologie, Bonn.

Engler, S. (1999). Hochschullehrer und die Herstellung von Geschlechtergrenzen. Der Empfang von Studentinnen und Studenten in Elektrotechnik und Erziehungswissenschaft. In Aylâ Neusel \& Angelika Wetterer (Hrsg.), Vielfältige Verschiedenheiten: Geschlechterverhältnisse in Studium, Hochschule und Beruf(S. 107-132). Frankfurt am Main; New York: Campus Verlag.

European Commission (2006). She Figures 2006. Luxemburg: Office for Official Publications of the European Communities.

Geenen, E. M. (1994). Blockierte Karrieren. Frauen in der Hochschule. In W. Röhrich \& C. Schlüter-Knauer (Hrsg.), Kieler Beiträge zur Politik und Sozialwissenschaft. Band 9. Opladen: Leske und Budrich.

Geenen, E. M. (2000). Akademische Karrieren von Frauen an wissenschaftlichen Hochschulen. In B. Krais (Hrsg.), Wissenschaftskultur und Geschlechterordnung: über die verborgenen Mechanismen männlicher Dominanz in der akademischen Welt (S. 83-106). Frankfurt/ Main; New York : Campus-Verlag

Gunter, R. \& Stambach, A. (2005). Differences in men and women scientist's perceptions of workplace climate. Journal of Women and Minorities in Science and Engineering, 11, 97116.

Haffner, Y., Könekamp, B. \& Krais, B. (2006). Arbeitswelt in Bewegung. Chancengleichheit in technischen und naturwissenschaftlichen Berufen als Impuls für Unternehmen. Hrsg. vom Bundesministerium für Bildung und Forschung (BMBF). Bonn

Hartmann, M. (2005): Eliten und das Feld der Macht. In C. Colliot-Thélène, F. Etienne, \& G. Gebauer (Hrsg.), Pierre Bourdieu: deutsch-französische Perspektiven (S. 255-275). Frankfur a.M.: Suhrkamp.

Heintz, B., Merz, M. \& Schumacher, C. (2004). Wissenschaft, die Grenzen schafft: Geschlechterkonstellationen im disziplinären Vergleich. Bielefeld: Transcript.

Heublein, U. \& Schwarzenberger, A. (2005). Studiendauer in zweistufigen Studiengängen - ein internationaler Vergleich. Hannover, Hochschul-Informations-System GmbH

Hoffmann-Lange, U. (2000). Umfrage über die Lebens- und Arbeitsbedingungen der Angehörigen des akademischen Mittelbaus sowie der Doktorandinnen/Doktoranden und Habilitandinnen/Habilitanden an der Universität Bamberg. Unter Mitarbeit von A. Rupprecht, S. Idelberger, E. Pfnür, T. Preunkert, S. Wagner. Unveröff. Manuskript, Otto-Friedrich Universität Bamberg.

Holtkamp, R., Fischer-Bluhm, K. \& Huber, L. (1986). Junge Wissenschaftler an der Hochschule. Frankfurt a.M.: Campus.

Holzbecher, M. (1997). Einstellungen von Hochschullehrern und Hochschullehrerinnen zur Hochschule als Ort für Frauen. In H. Macha \& M. Klinkhammer (Hrsg), Die andere Stimme der Wissenschaft. Stimmen der Frauen an Hochschulen (S. 39-45). Bielefeld: Kleine Verlag.

Holzbecher, M., Küllchen, H., Löther, A. \& Goldtammer, A. (2002). Fach-und fakultätsspezifische Ursachen der Unterrepräsentation von Frauen bei Promotionen. Interdisziplinäres Zentrum für Frauen- und Geschlechterforschung der Universität Bielefeld.

Janson, K., Schomburg, H. \& Teichler, U. (2006). Wissenschaftliche Wege zur Professur oder ins Abseits? Strukturinformationen zu Arbeitsmarkt und Beschäftigung an Hochschulen in Deutschland und den USA. Kassel: INCHER, International Centre for Higher Education Research, http://www.gain-network.org

Kiegelmann, M. (2000). Habilitation. Anmerkungen aufgrund einer empirischen Erhebung. Hochschule Ost, 3-4, 39-46.

Kirschbaum, A., Noeres, D., Flaake, K. \& Fleßner, H. (2005). Promotionsförderung und Geschlecht. Zur Bedeutung geschlechtsspezifisch wirkender Auswahlprozesse bei der Förderung von 
Promotionen an niedersächsischen Hochschulen. Bibliotheks- und Informationssystem der Universität Oldenburg.

Koch, P. M. (1995). Doktorandinnen. Der Wille zur wissenschaftlichen Anerkennung. In I. Modelmog (Hrsg.), Frauenforschung interdisziplinär - Theorie, Band 2. (Zugel. Dissertation, Uni Hamburg 1994). Münster / Hamburg: LIT Verlag.

Kort-Krieger, U. (1995). HSP-II-Stipendiatinnen. Eine empirische Untersuchung der Motive und Erfahrungen von Stipendiatinnen an sieben bayerischen Universitäten. München: unveröffentlichtes Manuskript.

Kracke, B. \& Englich, B. (1996). Vorbereitung auf die Professur? Erfahrungen von studentischen Hilfskräften am Arbeitsplatz Universität. In B. Kracke, \& E. Wild (Hrsg.), Arbeitsplatz Hochschule (S. 47-72). Heidelberg: Mattes Verlag.

Krais, B. \& Krumpeter, T. (1997). Wissenschaftskultur und weibliche Karrieren. MPG-Spiegel 3, 31-35.

Krimmer, H. \& Zimmer, A. (2003). Karrierewege von Professorinnen an Hochschulen in Deutschland. Zeitschrift für Frauenforschung und Geschlechterstudien, 4, 21, 18-33. Bielefeld: Kleine-Verlag.

Leemann, R. J. (2002). Chancenungleichheiten im Wissenschaftssystem. Wie Geschlecht und soziale Herkunft Karrieren beeinflussen. Zürich: Rüegger.

Lind, I. (2004a). Aufstieg oder Ausstieg? Karrierewege von Wissenschaftlerinnen. - Ein Forschungsüberblick. (CEWS, Beiträge Frauen in Wissenschaft und Forschung Nr. 2, herausgegeben von B. Mühlenbruch). Bielefeld: Kleine Verlag.

Lind, I. (2004b). Balancierung von Wissenschaft und Elternschaft. Mythen und Fakten. CEWSnewsletter Nr. 26. Online: [http://www.cews.org/cews/files/82/de/news26.pdf].

Lind, I. (2004c). Evaluation des Lise Meitner-Habilitationsprogramms. In A. Löther (Hrsg.), Erfolg und Wirksamkeit von Gleichstellungsmaßnahmen an Hochschulen (S. 122-147). (CEWS, Beiträge Frauen in Wissenschaft und Forschung Nr. 3, herausgegeben von B. Mühlenbruch. Bielefeld: Kleine Verlag.

Lind, I. \& Löther, A. (2006). Evaluation der Förderinstrumente Berufungstraining und Coaching. In J. Dalhoff (Hrsg.), Anstoßzum Aufstieg - Karrieretraining für Wissenschaftlerinnen auf dem Prüfstand (S. 26-87). (CEWS, Beiträge Frauen in Wissenschaft und Forschung Nr. 4, herausgegeben von B. Mühlenbruch). Bielefeld: Kleine Verlag.

Malone, K. R., Nersessian, N. J. \& Newstetter, W. (2005). Gendert writ small: Gender enactments and gendered narratives about lab organizations and knowledge transmission in a biomedical engineering research setting. Journal of Women and Minorities in Science and Engineering, 11, 61-81.

Matthies, H., Kuhlmann, E., Oppen, M. \& Simon, D. (2001). Karrieren und Barrieren im Wissenschaftsbetrieb. Geschlechterdifferente Teilhabechancen in außeruniversitären Forschungseinrichtungen. Berlin: edition sigma.

Mesletzky, J., Oelkers, S. \& Pasero, U. (1995). Evaluation der besonders Frauen fördernden Maßnahmen des Hochschulsonderprogramms II. Herausgegeben von der Ministerin für Wissenschaft, Forschung und Kultur des Landes Schleswig-Holstein, Kiel.

Mischau, A., Daniels, J.; Lehmann, J., Petersen, K. (2004). Geschlecht und Fachkulturen in der Mathematik - Ergebnisse einer empirischen Studie an der Universität Bielefeld (IFF-Forschungsreihe). Interdisziplinäres Zentrum für Frauen- und Geschlechterforschung der Universität Bielefeld.

Morrison, Z., Bourke, M. \& Kelley, C. (2005). 'Stop making it such a big issue': Perceptions and experiences of gender inequality by undergraduates at a British University. Women's Studies International Forum 28, 150-162.

National Science Foundation, Division of Science Resources Statistics (2004): Gender Differences in the Careers of Academic Scientists and Engineers: A Literature Review. Arlington, VA: National Science Foundation 
Noeres, D. \& Kirschbaum, A. (Hrsg.). (2004). Promotionsförderung und Geschlecht. Ergebnisse einer empirischen Studie und Folgerungen für die Praxis. Carl von Ossietzky Universität, Oldenburg.

Online: [http://www.uni-oldenburg.de/zfg/docs/Text_Promofoe_Workshop.pdf].

Ostendorf, H. (2005): Steuerung des Geschlechterverhältnisses durch eine politische Institution: die Mädchenpolitik der Berufsberatung. Opladen: B. Budrich.

Perkins, J. A. (1973). The University as an Organisation. New York: McGraw-Hill

Pfister, G. (1986). Anpassung, Widerstand, Resignation? Probleme und Perspektiven promovierender Frauen. In B. Clemens, S. Metz-Göckel \& A. Neusel, \& B. Port (Hrsg.), Töchter der Alma Mater. Frauen in der Berufs- und Hochschulforschung (S. 167-192). Frankfurt / New York: Campus.

Plicht, H. \& Schreyer, F. (2002). Ingenieurinnen und Informatikerinnen. Schöne neue Arbeitswelt? IAB-Berichte. Analysen aus dem Institut für Arbeitsmarkt-und Berufsforschung der Bundesanstalt für Arbeit, Nr. 11. Nürnberg

Ratzer, B., Hnilica S., Knoll, B. \& Szalai, E. (2006). Erster Zwischenbericht Gender in die Lehre (GiL) . TU Wien.

Röbbecke, M. (2004). Schritt für Schritt hinein in die Wissenschaft - oder hinaus? In: M. Oppen, \& D. Simon (Hrsg.), Verharrender Wandel. Institutionen und Geschlechterverhältnisse (S. 311-341). Berlin: edtion sigma.

Röbbecke, M. \& Simon, D. (2001). Promovieren mit Stipendium: zweite Evaluation der Förderung des wissenschaftlichen Nachwuchses nach dem Nachwuchsförderungsgesetz (NaFöG) . Berlin: Wissenschaftszentrum Berlin für Sozialforschung.

Schaeper, H. \& Minks, K.-H. (1997). Studiendauer - eine empirische Analyse ihrer Determinanten und Auswirkungen auf den Berufseintritt. Hannover: Hochschul-Informations-System $\mathrm{GmbH}$

Schreyer, F. (1999). Studienfachwahl und Arbeitslosigkeit. Frauen sind häufiger arbeitslos gerade wenn sie ein ,Männerfach' studiert haben. IAB Kurzbericht. Aktuelle Analysen aus dem Institut für Arbeitsmarkt- und Berufsforschung der Bundesanstalt für Arbeit, Nr. 14. Nürnberg

Seaman, R., Nolan, K. \& Dwyer, S. C. (2001). Breaking the cycle: only 1,920 more years to equity. Journal of Women and Minorities in Science and Engineering, 7, 19-34.

Spies, K. \& Schute, M. (1999). Warum promovieren Frauen seltener als Männer? Psychologische Prädiktoren der Promotionsabsicht bei Männern und Frauen. Zeitschrift für Sozialpsychologie, 4, 229-245.

Stadler, H. (2005). Dualismus und Wissenschaft —- Physik als männliche Domäne. In M. Bidwell-Steiner \& W. Wozonig (Hrsg.), Die Kategorie Geschlecht im Streit der Disziplinen (S. 206-222). Band 1 der Reihe "Gendered Subjects». Innsbruck-Wien-München-Bozen: Studienverlag.

Statistisches Bundesamt (1992). Bildung und Kultur, Fachserie 11, Reihe 4.2 Prüfungen an

Hochschulen, Wiesbaden.

Stebut, N. von (2003). Eine Frage der Zeit? Zur Integration von Frauen in die Wissenschaft. Eine empirische Untersuchung der Max-Planck-Gesellschaft. Opladen: Leske + Budrich.

Stegmann, S. (2005). „... got the look!» - Wissenschaft und ihr Outfit. Eine kulturwissenschaftliche Studie über Effekte von Habitus, Fachkultur und Geschlecht. Münster: LIT Verlag.

Vogel, U. \& Hinz, C. (2004). Wissenschaftskarriere, Geschlecht und Fachkultur. Bewältigungsstrategien in Mathematik und Sozialwissenschaften. Bielefeld: Kleine Verlag.

Wetterer, A. (1994): Professionalisierung, soziale Schließung und berufsspezifische Konstruktionen der Geschlechterdifferenz. In R. H. Stein \& A. Wetterer (Hrsg.), Studierende und studierte Frauen: Ein ost-west-deutscher Vergleich (S. 21-46) Kassel: Jenior u. Pressler.

Wimbauer, C. (1999). Organisation, Geschlecht, Karriere. Fallstudien aus einem Forschungsinstitut. Opladen: Leske + Budrich. 
Wissenschaftsrat (1997). Empfehlungen zur Doktorandenausbildung und zur Förderung des Hochschullehrernachwuchses. Köln.

Wissenschaftsrat (1998). Empfehlungen zur Chancengleichheit von Frauen in Wissenschaft und Forschung. Köln.

Wissenschaftsrat (2001). Entwicklung der Fachstudiendauer an Universitäten von 1990. Köln.

Zimmermann, M. (1996). Wissenschaftliche Aspiration von Studierenden und ihre Bedeutung für die Förderung des wissenschaftlichen Nachwuchses. In B. Kracke, \& E. Wild (Hrsg.), Arbeitsplatz Hochschule. Überlegungen und Befunde zur beruflichen Situation und Förderung des wissenschaftlichen Nachwuchses (S. 19-45). Heidelberg: Mattes Verlag.

Schlagworte: Wissenschaft, Hochschule, Geschlecht, Statistik, Unterrepräsen$\operatorname{tanz}$, Karriere

\section{Les chances des femmes en science: une question de culture disciplinaire ? Analyses rétrospectives de la qualification et résultats actuels de la recherche}

\section{Résumé}

Dans le débat sur les femmes scientifiques il est très souvent argumenté que si les femmes occupent si peu de postes à plus grande responsabilité c'est parce qu'il manque des étudiantes et des jeunes femmes au seuil d'une carrière scientifique. Létude présentée dans cette contribution vérifie cet argument en analysant les chances qu'ont les hommes et les femmes d'accéder à une qualification scientifique durant leur carrière sur une période de 18 ans. Nous présentons brièvement la méthode de l'étude qui consiste en une analyse retrospective des carrières. Les résultats confirment la participation inégale des femmes à la qualification scientifique. La disparité ne s'explique pas par la sous-représentation des femmes aux échelons inférieurs de la carrière scientifique. Les analyses par discipline scientifique révèlent d'importantes différences qui ne sont pas prises en compte jusqu'à présent. Les résultats des analyses rétrospectives sont interprétés à la lumière des recherches actuelles sur les obstacles que rencontrent les femmes scientifiques et sur les effets des cultures disciplinaires. Les résultats nous amènent à remettre en cause des hypothèses largement répandues sur les raisons de la disparité des femmes et des hommes dans la science. En plus, l'analyse en fonction des différentes disciplines offre des nouvelles perspectives de recherche.

Mots clés: science, école supérieure, genre, statistique, sous-représentation, carrière 


\title{
Le opportunità per le donne nella scienza: una questione di cultura disciplinare? Analisi retrospettiva dei processi qualificativi e risultati delle ricerche più recenti
}

\author{
Riassunto
}

In questo contributo sarà analizzata per la prima volta e separatamente per i due generi, l'opportunità di svolgere una carriera scientifica sull'arco di 18 anni. A titolo introduttivo è presentato il metodo dell'analisi retrospettiva. I risultati ottenuti dimostrano la minore partecipazione al processo di qualifica scientifico da parte delle donne, tale partecipazione non è però riconducibile solo a determinati settori. Osservando più nel dettaglio, si possono rilevare anche grandi differenze tra discipline che finora non erano state evidenziate. Il sistema di analisi retrospettiva è completato dalle conoscenze attuali sulle barriere che rendono difficile l'accesso alla scienza per le donne e sull'effetto della cultura disciplinare. In base ai risultati riportati, vengono esaminate in modo critico le considerazioni diffuse sulla scarsa partecipazione delle donne nel campo scientifico, inoltre, sono poste delle nuove prospettive per la ricerca in questa direzione.

Parole chiave: Scienza, università, genere, statistica, sottorappresentazione, carriera

\section{Women and Science - a question of disciplinary cultures? - Retrospective analyses of the qualification and result of the latest research}

\section{Summary}

Based on available data, this paper analyzes, for the first time, the statistical probability for men and women in Germany holding a professorship after 18 years of scientific qualification process. After a brief introduction, the paper explains the method of retrospective data analysis following first year students in the 1980s on to new professorships in 2003. Specific examples are given to show the remarkable differences of female participation in the qualification process between the disciplines. These results are supplemented by knowledge drawn form the research body concerning the marginalization of women in science and the different disciplinary cultures. The main message is to critically reflect some widespread and partially misleading assumptions of the causes of the female marginalization in science and to give some new perspectives of discussion and research desiderata.

Keywords: Science, University, gender, under-representation, scientific career 